\title{
Solution-processed zinc oxide field-effect transistors based on self-assembly of colloidal nanorods
}

\author{
Baoquan Sun and Henning Sirringhaus*
}

Cavendish Laboratory, University of Cambridge, Madingley Road, Cambridge, CB3 OHE United Kingdom

Supporting information

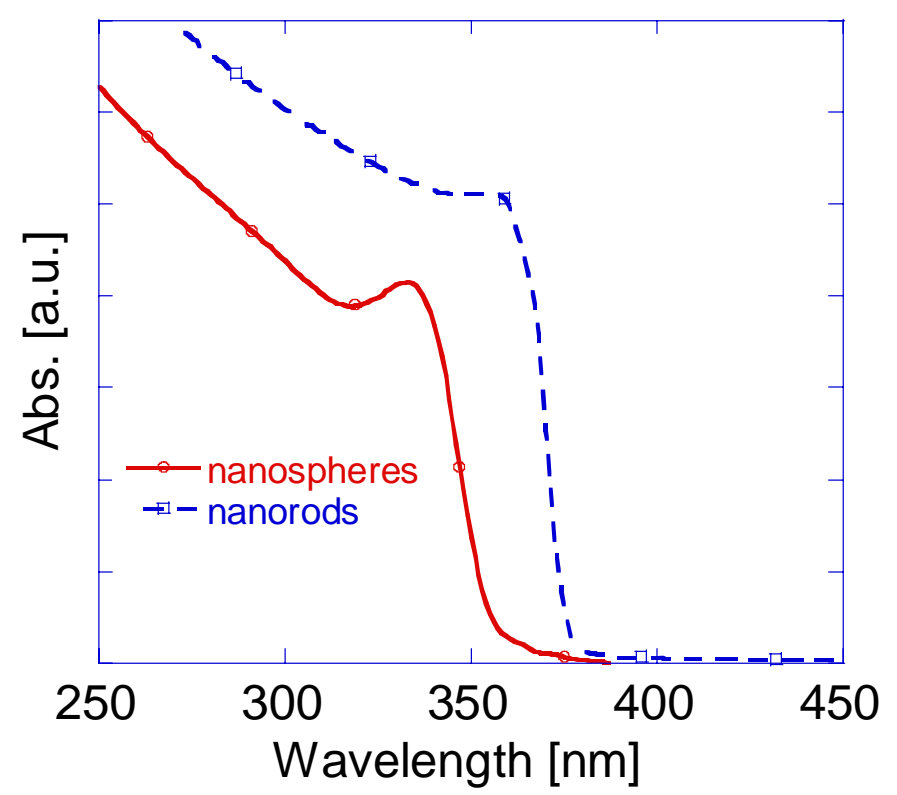

Figure 1S: Absorption spectrum of $\mathrm{ZnO}$ nanospheres and nanorods dispersed in chloroform/methanol solution. The absorption peak is shifted from $330 \mathrm{~nm}$ (nanospheres) to 360 $\mathrm{nm}$ (nanorods) reflecting the increased diameter of the nanorods. 


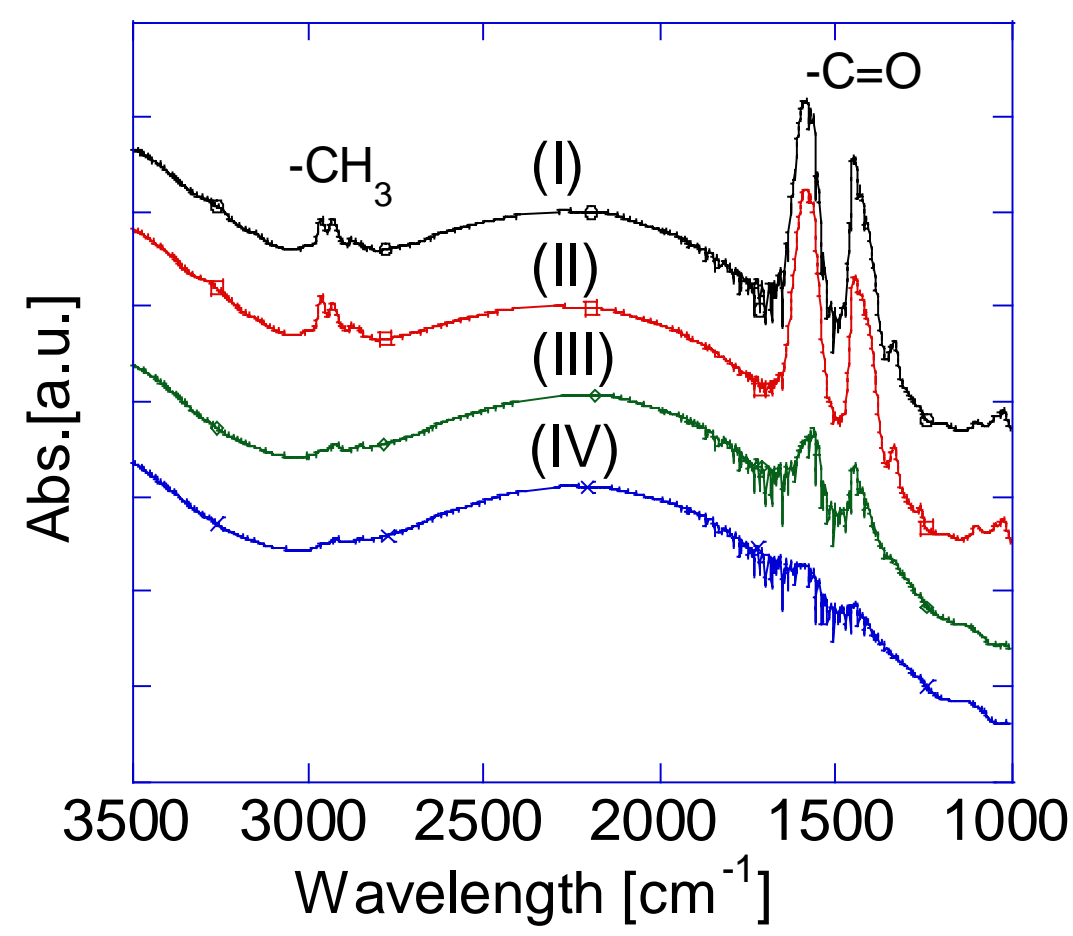

Figure 2S FTIR spectrum of $\mathrm{ZnO}$ nanocrystal films: (I) $\mathrm{ZnO}$ with acetate ligand only after spin-coating; (II) $\mathrm{ZnO}$ with added butylamine ligand after spin-coating; (III) $\mathrm{ZnO}$ with acetate group only after annealing at $230{ }^{\circ} \mathrm{C}$; IV) $\mathrm{ZnO}$ with added butylamine ligand after annealing at $230{ }^{\circ} \mathrm{C}$. The sample preparation and measurements are all performed in nitrogen atmosphere to avoid effects due to $\mathrm{H}_{2} \mathrm{O}$ and $\mathrm{CO}_{2}$. 


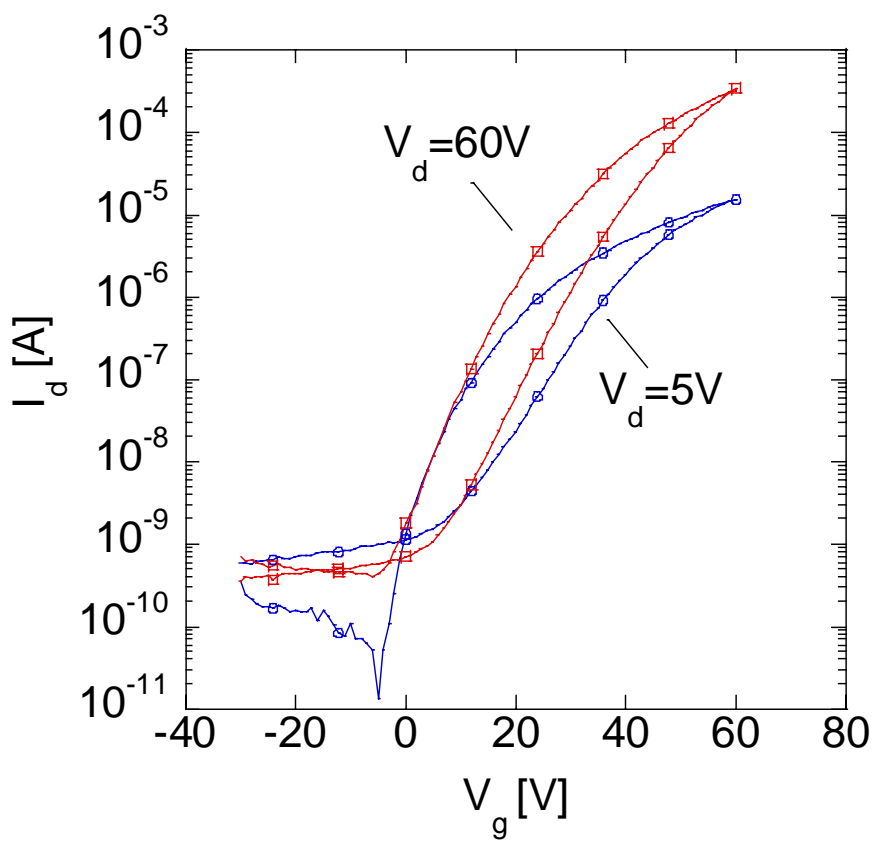

Figure 3S Transfer characteristics of a TFT device made from nanorods after hydrothermal growth but without further annealing in hydrogen atmosphere. The device is measured in nitrogen. 


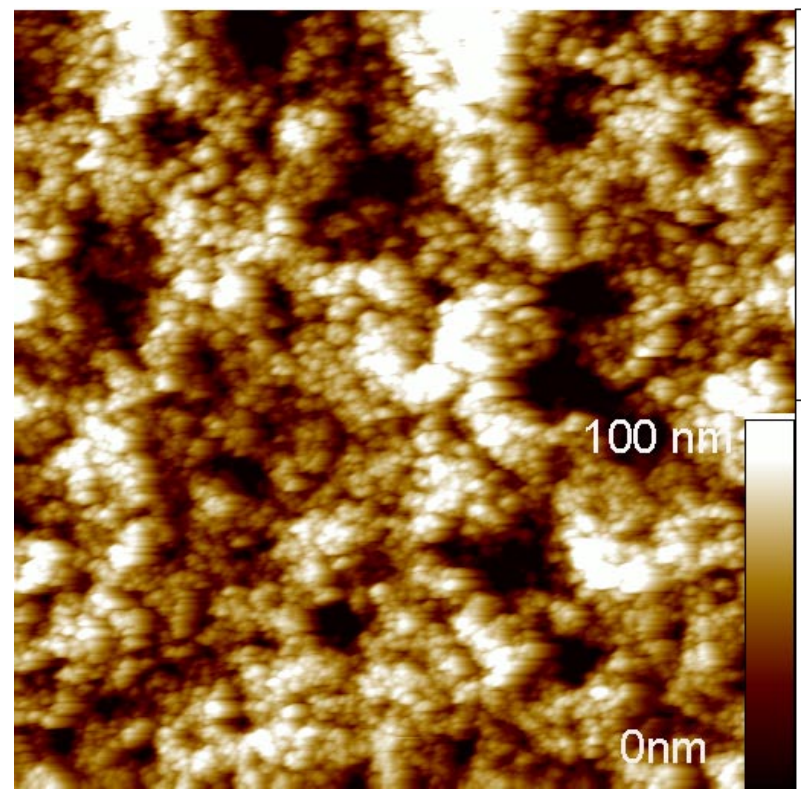

Figure 4S Large-area atomic force microscope topography image of an as-spin-coated nanorod film. The scanning range is $5 \mu \mathrm{m} \times 5 \mu \mathrm{m}$, the $\mathrm{z}$ topography scale is shown on the right.

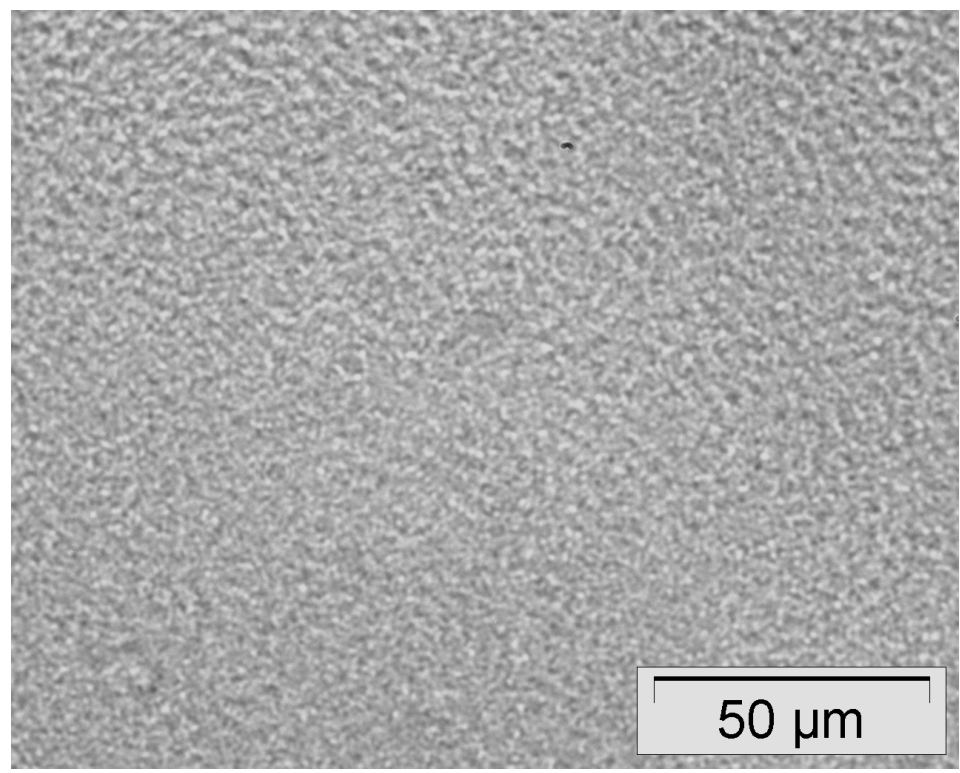

Figure 5S Optical microscope image showing the uniformity of an as-spin-coated nanorod film. 